\title{
Properties of generalized synchronization in smooth and non-smooth identical oscillators
}

\author{
M. Balcerzak, A. Chudzik, and A. Stefanski ${ }^{\mathrm{a}}$ \\ Division of Dynamics, Lodz University of Technology, ul. Stefanowskiego 1/15 Lodz, \\ Poland
}

Received 24 January 2020 / Accepted 8 June 2020

Published online 28 September 2020

\begin{abstract}
This paper deals with the phenomenon of the GS only in the context of unidirectional connection between identical exciter and receivers. A special attention is focused on the properties of the GS in coupled non-smooth Chua circuits. The robustness of the synchronous state is analyzed in the presence of slight parameter mismatch. The analysis tools are transversal and response Lyapunov exponents and fractal dimension of the attractor. These studies show differences in the stability of synchronous states between smooth (Lorenz system) and non-smooth (Chua circuit) oscillators.
\end{abstract}

\section{Introduction}

In analysis and theory of dynamical systems, the research of interactions between them plays an important role. Such interactions often lead to an appearance of some synchronization effects. First time this phenomenon has been observed by C. Huygens in the second half of 17 th century [1] and it was [2-4] and still is actively studied by many researchers to this day.

Especially interesting are oscillators exhibiting chaotic or stochastic dynamics. In recent years, the chaotic synchronization has become an object of great interest in many areas of science [5-11]. Consequently, a number of new types of synchronization have been also identified, e.g., generalized synchronization $[12,13]$, phase [14-16] and imperfect phase synchronization $[17,18]$, anticipated synchronization $[19,20]$ and ragged synchronizability [21].

One of the most interesting proposals appearing in the context of chaos synchronization, is a concept called the generalized synchronization (GS). This term has been introduced by Rulkov et al. [12] as a generalization of the synchronization idea for unidirectionally coupled systems:

$$
\begin{gathered}
\dot{x}=f(x), \\
\dot{y}=g(x, y),
\end{gathered}
$$

a e-mail: andrzej.stefanski@p.lodz.pl 


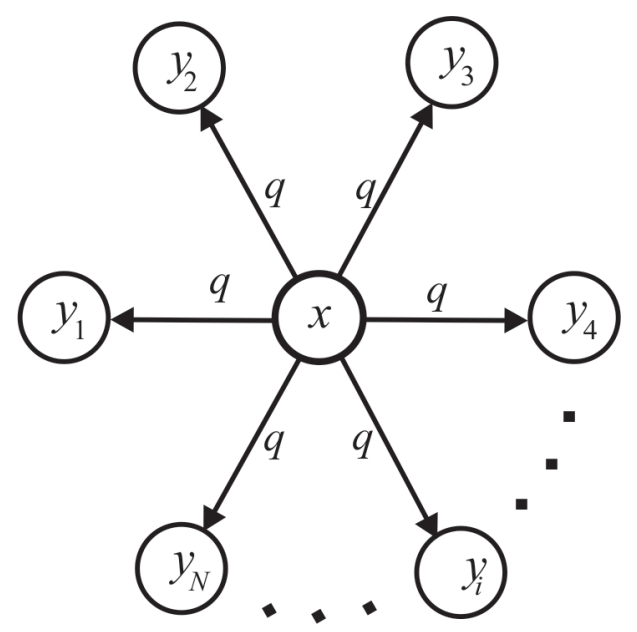

Fig. 1. Common drive - a star configuration with a unidirectional coupling.

where $x \in \mathbb{R}^{m}, y \in \mathbb{R}^{k}$. Such unidirectional link of dynamical systems is also called the master-slave coupling. We can say that the GS of these systems occurs if there exists a static functional relation $\psi$ between their states, i.e.,

$$
y(t)=\psi[x(t)]
$$

In general, the GS phenomenon has been considered both in the context of identical (when separated) systems (1a) and (1b), and also in cases when the response system is slightly (the same set of ODEs with different values of system parameters) or strictly different (another set of ODEs and attractor dimension) than the driving oscillator [13,22-24]. This phenomenon can be also observed in discrete time systems [25-28]. However, in any of these cases the phenomenon of complete synchronization (CS) can be applied as a tool for recognizing the GS. In classical papers on the GS Pyragas distinguished [25,26] two types of this phenomenon, considering the properties of synchronization manifold, i.e., the weak and strong GS.

In order to identify the GS, a method called the mutual false nearest neighbors (Rulkov et al. [12]) and the related auxiliary system approach (Abarbanel et al. [22]) can be applied. According to them, the criterion for the GS existence is an appearance of the CS between the response system [Eq. (1b)] and its identical duplication, i.e.,

$$
\lim _{t \rightarrow \infty}\left\|y\left(t, x_{0}, y_{01}\right)-y\left(t, x_{0}, y_{02}\right)\right\|=0
$$

where $\left(x_{0}, y_{01}\right)$ and $\left(x_{0}, y_{02}\right)$ are two initial conditions of systems (1a) and (1b). An occurrence of such CS [Eq. (3)] indicates that slave systems forget their initial states and then the functional control defined by equation (2) takes place. Thus, the GS detection can be treated as the equivalent of the synchronization of a series of dynamical systems under the influence of common external drive. In such a startype connections between oscillators, a unidirectional coupling from the central node (exciter $e$ ) to the remaining oscillators (receivers $x_{i}, i=1,2, \ldots, N$ ) is realized, as shown schematically in Figure 1.

In recent years the idea of the GS has been developed for mutually coupled systems $[29,30]$ and even for networks of coupled oscillators [30-32]. Consequently, the technique of auxiliary system approach has been also adopted to these cases [33]. Moreover, same new aspects of the GS phenomenon have been observed and 
described, e.g., effect of noise [34], routes to the CS [35], passive, resonant and interactive GS [36], an analytic criterion for the GS [37] and some new aspects of the weak and strong GS [38].

This paper deals with the phenomenon of the GS only in the context of unidirectional connection between identical exciter and receivers. A special attention is focused on the properties of the GS in coupled non-smooth Chua circuits. The robustness of the synchronous state is analyzed in the presence of slight parameter mismatch. The analysis tools are transversal and response Lyapunov exponents and fractal dimension of the attractor. These studies show differences in the stability of synchronous states between smooth (logistic map, Lorenz system) and non-smooth (Chua circuit) oscillators. On the basis of these observations new criterion for the strong GS has been proposed.

The paper is organized as follows. Section 2 contains detailed description of tools for the system dynamics analysis that have been used during the study. In Section 3 results of numerical investigation of the GS phenomenon are demonstrated. Finally, Section 4 presents a discussion of the results and conclusions.

\section{Detection of the generalized synchronization}

Consider equations (1a)-(1b) in matrix form of unidirectionally coupled identical systems:

$$
\begin{aligned}
& \dot{x}=f(x), \\
& \dot{y}=f(y)+q[h(x)-h(y)],
\end{aligned}
$$

where $q$ is coupling coefficient and $h(x): \mathbb{R}^{m} \rightarrow \mathbb{R}^{k}$ is a function characterizing the coupling between the drive (Eq. (1a)) and the response (Eq. (1b)) system - linking function.

The strong GS of identical systems in the master-slave configuration manifests with the CS between the drive (Eq. (1a)) and the response (Eq. (1b)) subsystems [25]. Then the functional relation given by equation (2) assumes the form of equality $y(t)=x(t)$. The best tools for testing the stability of such synchronous state are so-called transversal Lyapunov exponents (TLEs). These quantities determine the divergence of nearby trajectories in directions transverse to the synchronization manifold. Therefore, it requires separating the transverse modes from the longitudinal ones in the variational equation. Differentiating system (4) we get

$$
\begin{aligned}
& \delta \dot{x}=D f(x) \delta x, \\
& \delta \dot{y}=D f(x) \delta x+q D h(x)(\delta x-\delta y),
\end{aligned}
$$

where $\delta x$ and $\delta y$ represent the perturbation of the drive and response systems, respectively, $D f(x)$ is the Jacobi matrix of them (assuming synchronous state $x=y$ ), and $D h(x)$ is the Jacobian of the linking function $h(x)$.

The next stage is a diagonalization of equation (5). Such block diagonalization leads to the uncoupling of variational equations (5) into blocks like in a mode analysis. After such a block diagonalization of the variational equation, there appear two separated blocks:

$$
\delta \dot{x}_{k}=\left[D f(x)+q \gamma_{k} D h(x)\right] \delta x_{k},
$$

where $\delta x_{k}$ represents different modes of a perturbation from the synchronous state $\left(\delta x_{0}=\delta x, \delta x_{1}=\delta y\right)$ and $\gamma_{k}$ represents a $k$ th eigenvalue of the connectivity matrix, 
$k=0,1$. For $k=0$ we have $\gamma_{0}=0$ and equation (6) is reduced to the variational equation of the separated driving system:

$$
\delta \dot{x}=D f(x) \delta x
$$

corresponding to the longitudinal direction located within the synchronization manifold. The second eigenvalue $\gamma_{1}=-1$ corresponds to the transverse eigenvector. Consequently, the generic variational equation for calculation of TLEs is now as follows:

$$
\delta \dot{x}=[D f(x)-q D h(x)] \delta x .
$$

Obviously, the calculation of TLEs requires a simultaneous integration of the reference system (1a).

In general, the TLEs calculated on the basis of equation (8) are a precise criterion for stability of the synchronous state (the strong GS) of identical systems coupled in master-slave relationship. Such a connection often causes the transversal (with respect to the synchronization manifold) convergence of trajectories in the phase space. Hence the condition for the strong GS of identical master-slave systems is

$$
\lambda_{j}^{T}<0
$$

However, in the case of externally driven oscillators, the mechanism of synchronization is slightly different. Namely, the synchronization of response oscillators is possible if they "forget their initial conditions" [23]. Such a situation takes place when the Lyapunov exponents characterizing response subsystems are negative. Therefore, these exponents have been called response Lyapunov exponents or (RLEs). The RLEs are often consider as an equivalent and even analogy to the conditional Lyapunov exponents (CLEs) concept.

In order to investigate the synchronizability of the array of externally excited oscillators, the properties of the GS, mentioned in Section 1, have been employed [12, 13,22]. We have assumed that all response oscillators are identical. On the other hand, the central driving oscillator (exciter $x$, see Fig. 1) may differ from response oscillators not only by the value of parameters but also by the form of differential equations. The dynamics of the entire system of commonly driven oscillators is expressed by the following equations:

$$
\begin{gathered}
\dot{x}=f(x), \\
\dot{\mathbf{y}}=\mathbf{G}(\mathbf{y})+q[\mathbf{I} \otimes h(x)],
\end{gathered}
$$

where $\mathbf{y}=\left(y_{1}, y_{2}, \ldots, y_{N}\right), \mathbf{G}(\mathbf{y})=\left[g\left(y_{1}\right), \ldots, g\left(y_{N}\right)\right]$ and $\mathbf{I}$ is the unit matrix.

The solution to individual $i$ th response oscillator of the system (10a)-(10b) exists in the $(k+m)$-dimensional phase space $\boldsymbol{X} \oplus \boldsymbol{Y}$, where $\boldsymbol{X}$ is a $k$-dimensional driving system phase space (Eq. (10a)) and $\boldsymbol{Y}$ is the phase space ( $m$-dimensional response subspace) of the individual response system (Eq. (10b)). The solution is characterized by a spectrum of a $k$-number of driving Lyapunov exponents (DLEs) and an $m$ number of the RLEs. The solution of the response system (Eq. (10b)) can be assumed in the following form:

$$
y_{i}(t)=\phi\left[t, y_{i}(0)\right]+\psi[x(t)]
$$


where $\phi$ and $\psi$ represent the functional parts of the solution, which are dependent on and independent of the response sub-system, respectively. In order to examine the synchronization tendency of the response oscillators, let us consider two of them, arbitrarily chosen from system (10b), i.e., $y_{i}$ and $y_{i+1}$. The time evolution of the trajectory separation between them (synchronization error) is described by the equation:

$$
\dot{y}_{i}-\dot{y}_{i+1}=g\left(x, y_{i}\right)-g\left(x, y_{i+1}\right) \text {. }
$$

The linearization of equation (12) leads to the following generic variational equation:

$$
\delta \dot{y}=D g[x(t), y(t)] \delta y,
$$

where $y$ represent each $i$ th response subsystem and $D g[x(t), y(t)]$ is its Jacobi matrix. On the basis of equation (13), the RLEs of systems (10a) and (10b) can be calculated. From equations (12) and (13) it results that the synchronization error tends to zero and the synchronous state is stable if all the RLEs are negative:

$$
\lambda_{j}^{R}<0
$$

where $j=1,2, \ldots, m$. Then, the component of solution (11) associated with the response of the system $\phi[t, y(0)]$ tends to zero and there appears a functional relation between the drive and the response systems analogous to equation (2). Thus, the GS of systems (10a) and (10b) takes place because the external drive results in the response, forgetting its initial condition. This type of the synchronous relationship is called the weak GS [25].

In general the TLE and the RLE are sufficient criteria for detection of the strong and the weak GS, respectively, in case of identical drive-response systems. However, the qualitative structural (different equation of motion) or even quantitative (different values of parameters) difference between them prevents use of TLEs as a criterion for the strong GS. Also the use of RLEs as the weak GS criterion is uncertain. Therefore, classical criteria based on the attractor dimension have been introduced [25,26]. The properties of the synchronization manifold allow us to define types of the GS as follows:

(i) the weak GS characterized by non-smooth mapping given by equation (2), which is observed when the global dimension of the strange attractor $d^{G}$, located in the whole phase space $\boldsymbol{X} \oplus \boldsymbol{Y}$, is larger than the attractor dimension of the driving system $d^{D}$, i.e.,

$$
d^{G}>d^{D}
$$

(ii) the strong GS, defining by the equality

$$
d^{G}=d^{D}
$$

i.e., the response oscillator does not influence the global attractor. Then the relation $y(t)=\psi[x(t)]$ is smooth. The dimension of attractors can be determined with use of the Lyapunov exponents spectrum according to the Kaplan \& Yorke formula [39]:

$$
d_{L}=j+\frac{\sum_{1}^{j} \lambda_{i}}{\left|\lambda_{j+1}\right|},
$$

where $j$ is the largest integer number for which the inequality $\sum_{1}^{j} \lambda_{i} \geq 0$ is fulfilled. 


\section{Numerical studies}

In this section, the results of numerical investigations of the weak and strong GS are demonstrated. In order to demonstrate the GS synchronization properties, three examples of standard chaotic systems in a master-slave configuration of the coupling have been considered. They are diffusively and unidirectionally coupled maps (Eqs. (18a)-(18c)) or flows (Eqs. (19a)-(19c)), described in the general form as follows:

$$
\begin{gathered}
x_{n+1}=f\left(x_{n}\right), \\
y_{n+1}=f\left(y_{n}\right)+q\left[f\left(x_{n}\right)-f\left(y_{n}\right)\right], \\
y_{n+1}^{\prime}=f\left(y_{n}^{\prime}\right)+q\left[f\left(x_{n}\right)-f\left(y_{n}^{\prime}\right)\right],
\end{gathered}
$$

or

$$
\begin{gathered}
\dot{x}=f(x), \\
\dot{y}=f(y)+q(x-y), \\
\dot{y}^{\prime}=f\left(y^{\prime}\right)+q\left(x-y^{\prime}\right),
\end{gathered}
$$

where $y^{\prime}$ represents an auxiliary response system for detecting the weak GS between drive and response oscillators. As has been mentioned in previous Section 2, the GS criteria for a particular case of completely identical drive-response systems are TLEs and RLEs. However, in such case the GS can be also simple detected in direct simulations of the synchronization process in systems (18a)-(18c) or (19b)-(19c). Namely, the strong GS means the CS between the master system (18a) or (19a) and response oscillators $(18 \mathrm{~b})-(18 \mathrm{c})$ or $(19 \mathrm{a})-(19 \mathrm{c})$. On the other hand, the weak GS occurs when the CS of the response $y$ and its auxiliary replica $y^{\prime}$ can be observed, whereas their synchronized motion remains uncorrelated with the drive $x$.

\subsection{Logistic maps}

As the first example, let us consider one-dimensional logistic maps introduced into equations (18a)-(18c):

$$
\begin{gathered}
x_{n+1}=a x_{n}\left(1-x_{n}\right), \\
y_{n+1}=(1-q)\left[a y_{n}\left(1-y_{n}\right)\right]+q\left[a x_{n}\left(1-x_{n}\right)\right], \\
y_{n+1}^{\prime}=(1-q)\left[a y_{n}^{\prime}\left(1-y_{n}^{\prime}\right)\right]+q\left[a x_{n}\left(1-x_{n}\right)\right] .
\end{gathered}
$$

Analogous classical examples are well known in the literature of this topic, e.g., references [25-27], but we present it in context of comparison with remaining results 


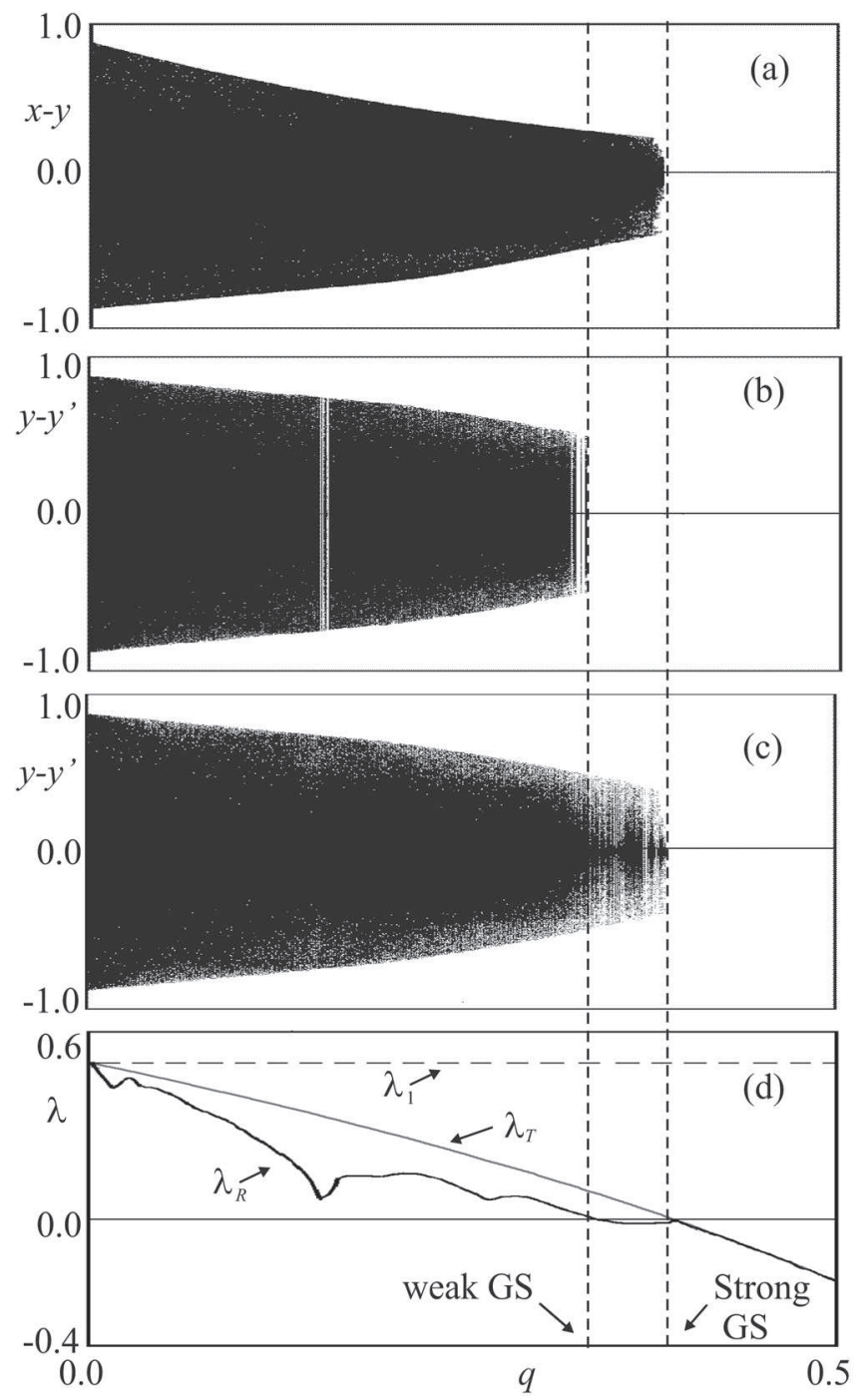

Fig. 2. Bifurcation diagrams of the synchronization error versus the coupling coefficient $q$ in the system of unidirectionally coupled logistic maps (Eqs. (20a)-(20c)). (a) Identical drive response oscillators $x-y$, (b) identical response oscillators $y-y^{\prime}$, (c) slightly different response oscillators $y-y^{\prime}$, i.e., $\Delta a=a \times 10^{-12}$, (d) corresponding Lyapunov exponents of system (20a)-(20c): the driving LE $\left(\lambda_{1}\right)$ - dashed line, the TLE $\left(\lambda_{T}\right)$ - gray curve, the RLE $\left(\lambda_{R}\right)$ - black line. Parameter $a=3.9$.

of our research. The numerical analysis of various synchronization aspects in system (20a)-(20c) is graphically depicted in Figures $2 \mathrm{a}-2 \mathrm{~d}$, where bifurcation diagrams of the trajectory separation (Figs. 2a-2c) and corresponding Lyapunov exponents (Fig. 2d) versus the coupling parameter $q$ are presented. Here, dashed vertical lines mark the thresholds the weak and strong GS, respectively. Comparing the diagrams from Figures $2 \mathrm{a}$ and $2 \mathrm{~b}$, we can see that the weak GS $\left(y-y^{\prime}\right.$ in Fig. $\left.2 \mathrm{~b}\right)$ appears below the threshold of the strong one $(x-y$ in Fig. 2a). Such a weakness of the GS is confirmed by the bifurcation diagram shown in Figure 2c, which has been evaluated for marginally different response oscillators, i.e., the mismatch of parameter 
$a$ between the response systems $y$ and $y^{\prime}$ amounts $\Delta a=a \times 10^{-12}$. However, even such an "invisible" parameter disturbance causes a destruction of the weak synchrony regime. Then, the synchronization threshold is determined by the imperfect complete synchronization (ICS) between $y$ and $y^{\prime}$. The comparison of pictures Figures 2a and 2c demonstrates that this ICS limit parameter value approximately coincides with the strong GS threshold. Thus, it reflects the properties of weak and strong versions of the GS. On the other hand, a quantitative criterion for both cases of the GS is given by the spectrum of three Lyapunov exponents of triple system (20a)-(20c) shown in Figure 2d. The first of them $\lambda_{1}$ (dashed horizontal line) is the largest LE of the driving oscillator (20a), which is obviously independent of the coupling parameter. Therefore, it has a constant value in spite of the increasing coupling strength. The second one (gray curve) is the TLE of the identity manifold $x=y$ :

$$
\lambda_{T}=\ln (1-q)+\lim _{n \rightarrow \infty} \frac{1}{n} \sum_{i=1}^{n} \ln \left|f\left(x_{i}\right)\right|,
$$

which in the case under consideration is reduced to the form:

$$
\lambda_{T}=\ln (1-q)+\lambda_{1}
$$

According to the assumed criteria, a negativity of $\lambda_{T}$ is the condition for the strong GS. The irregular black line in Figure 2d represents the RLE $(\lambda R)$ of the drive-response system (20a)-(20b), which is defined by the formula:

$$
\lambda_{T}=\ln (1-q)+\lim _{n \rightarrow \infty} \frac{1}{n} \sum_{i=1}^{n} \ln \left|f\left(y_{i}^{\prime}\right)\right| .
$$

From the comparison of Figures $2 \mathrm{~b}$ and $2 \mathrm{~d}$, it results that the weak GS in system $((20 \mathrm{a})-(20 \mathrm{c}))$ takes place if the largest $\operatorname{RLE}\left(\lambda_{R}\right)$ is negative in the coupling parameter space.

\subsection{Lorenz oscillators}

The second example is chaotic Lorenz oscillators [40] coupled via first variables according to equations (19a)-(19c):

$$
\begin{gathered}
\dot{x}_{1}=-\sigma x_{1}+\sigma x_{2}, \\
\dot{x}_{2}=-x_{1} x_{3}+r x_{1}-x_{2}, \\
\dot{x}_{3}=x_{1} x_{2}-b x_{3}, \\
\\
\dot{y}_{1}=-\sigma y_{1}+\sigma y_{2}+q\left(x_{1}-y_{1}\right), \\
\dot{y}_{2}=-y_{1} y_{3}+r y_{1}-y_{2}, \\
\dot{y}_{3}=y_{1} y_{2}-b y_{3}, \\
\\
\dot{y}_{1}^{\prime}=-\sigma y_{1}^{\prime}+\sigma y_{2}^{\prime}+q\left(x_{1}-y_{1}^{\prime}\right), \\
\dot{y}_{2}^{\prime}=-y_{1}^{\prime} y_{3}^{\prime}+r y_{1}^{\prime}-y_{2}^{\prime}, \\
\dot{y}_{3}^{\prime}=y_{1}^{\prime} y_{2}^{\prime}-b y_{3}^{\prime} .
\end{gathered}
$$


The Jacobi matrices for calculation of the TLEs (Eq. (8)) and RLEs (Eq. (13)), respectively, are as follows

$$
\begin{aligned}
{[D f-q D h(x)] } & =\left(\begin{array}{ccc}
-\sigma-q & \sigma & 0 \\
-x_{3}+r & -1 & -x_{1} \\
x_{2} & x_{1} & -b
\end{array}\right) \quad \text { and } \\
D g[t, y(0)] & =\left(\begin{array}{ccc}
-\sigma-q & -\sigma & 0 \\
-y_{3}+r & -1 & -y_{1} \\
y_{2} & y_{1} & -b
\end{array}\right)
\end{aligned}
$$

The results of the numerical analysis of the synchronous behavior in system (24a)(24c) are demonstrated in Figures 3a-3e. The synchronization errors have been calculated as a phase distance of system's trajectories, i.e.,

$$
|x-y|=\sqrt{\left(x_{1}-y_{1}\right)^{2}+\left(x_{2}-y_{2}\right)^{2}+\left(x_{3}-y_{3}\right)^{2}}
$$

and

$$
\left|y-y^{\prime}\right|=\sqrt{\left(y_{1}-y_{1}^{\prime}\right)^{2}+\left(y_{2}-y_{2}^{\prime}\right)^{2}+\left(y_{3}-y_{3}^{\prime}\right)^{2}} .
$$

We can observe a full analogy to the case of discrete-time systems (20a)-(20c). The thresholds of the weak ( $q=24.6$, see Fig. 3a) and strong $(q=35.7$, see Fig. 3b) GS are determined by the negativity of the largest RLE and TLE (Fig. 3e), respectively. Moreover, an introduction of the small parameter mismatch, i.e., a perturbation of the control parameter $r$ in the response Lorenz systems $y$ and $y^{\prime}$ is $\Delta r=r \times 10^{-4}$, also causes a desychronous effect (Fig. 3c), similarly to the logistic map case (Fig. 2c). Thus, it is a proof that the weak GS regime is very sensitive to the mismatch of response oscillators. On the other hand, after crossing the strong GS threshold at $q=35.7$, imperfect synchronous regimes $x-y$ and $y-y^{\prime}$ remain stable even in the presence of small parameter mismatch (see Figs. 3c and 3d). These results show that a real condition for the strong GS of identical drive-response systems is $\lambda_{T}^{1}<0$, in spite of the fact that the dimension of the global attractor is still larger than the dimension of the driving system attractor, i.e., the strong GS condition (Eq. (16)) is not fulfilled. For instance, the dimension of the driving system (24a) attractor is $d^{D}=2.101$, while the global dimension of the drive-response system (24a)-(24b) is $d^{G}>4$ even in the range $\lambda_{T}^{1}<0$, where the strong GS takes place (see Figs. 3a, 3c and $3 \mathrm{~d}$ ). Consequently, the sufficient GS condition in the case under consideration (Eqs. (24a)-(24c)) is negativity of the TLEs.

\subsection{Chua circuits}

The last of considered cases of master-slave coupling configuration (Eqs. (19a)-(19c)) are non-smooth Chua circuits [41] connected via second variables:

$$
\begin{aligned}
& \dot{x}_{1}=\alpha\left\{x_{2}-x_{1}-\left[m_{0} x_{1}+0.5\left(m_{1}-m_{0}\right)\left(\left|x_{1}+1\right|-\left|x_{1}-1\right|\right)\right]\right\} \\
& \dot{x}_{2}=x_{1}-x_{2}+x_{3} \\
& \dot{x}_{3}=-\beta x_{2} \\
& \dot{y}_{1}=\alpha\left\{y_{2}-y_{1}-\left[m_{0} y_{1}+0.5\left(m_{1}-m_{0}\right)\left(\left|y_{1}+1\right|-\left|y_{1}-1\right|\right)\right]\right\} \\
& \dot{y}_{2}=y_{1}-y_{2}+y_{3}+q\left(x_{2}-y_{2}\right) \\
& \dot{y}_{3}=-\beta y_{2}
\end{aligned}
$$




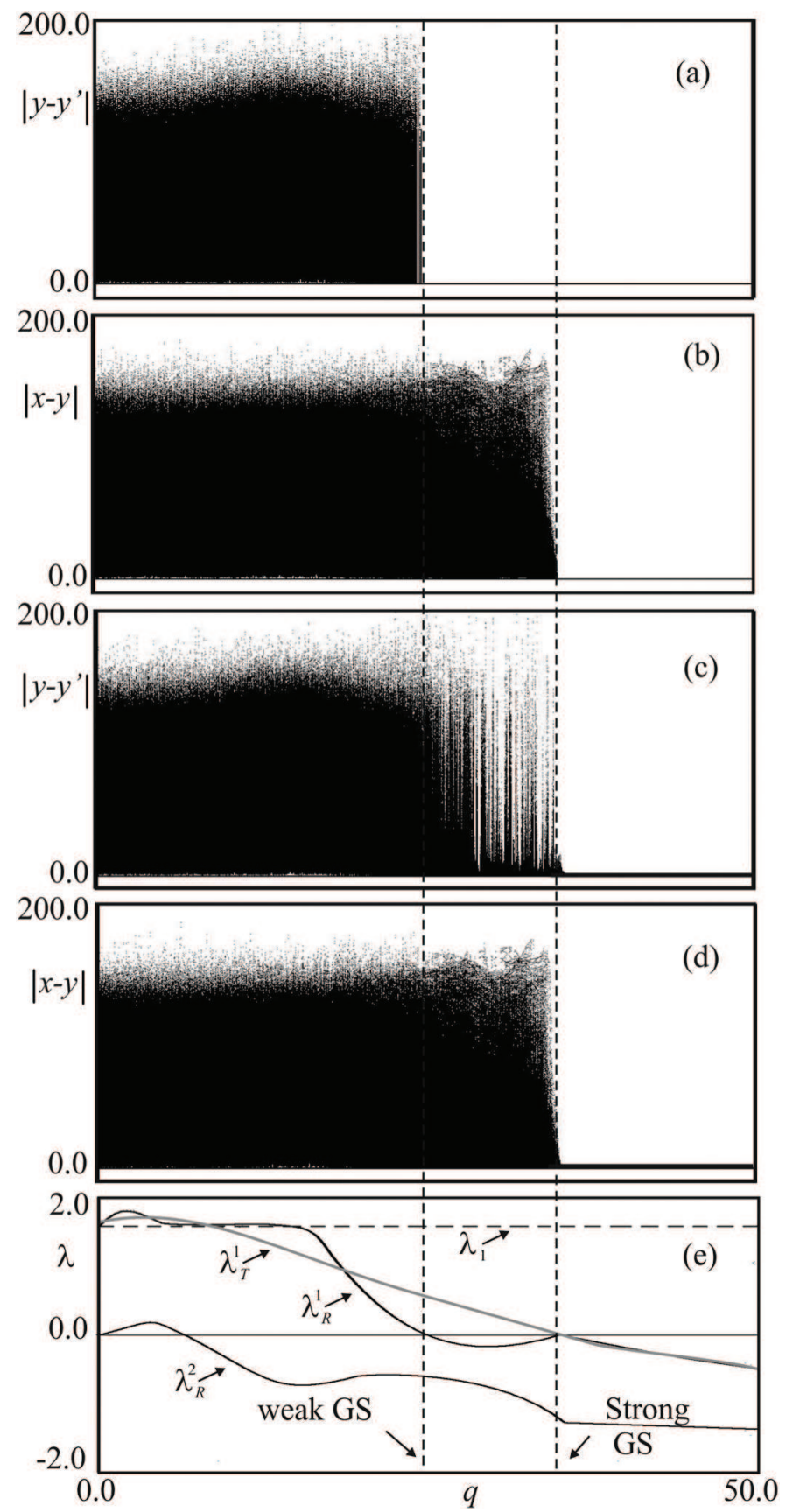

Fig. 3. Bifurcation diagrams of the synchronization error versus the coupling coefficient $q$ in the system of unidirectionally coupled Lorenz oscillators (Eqs. (24a)-(24c)). (a) Identical response oscillators $y-y^{\prime}$, (b) identical drive-response systems $x-y$, (c) slightly different response oscillators $y-y^{\prime}: \Delta r=r \times 10^{-4}$, (d) slightly different drive and response oscillators $x-y: \Delta r=r \times 10^{-4}$, (e) corresponding Lyapunov exponents of system (2a-2c): the driving LE $\left(\lambda_{1}\right)$ - dashed line, the largest TLE $\left(\lambda_{T}^{1}\right)$ - gray curve, and two the largest $\operatorname{RLE}\left(\lambda_{R}^{1}\right.$, $\left.\lambda_{R}^{2}\right)$ - black line. System parameters: $\sigma=10.0, b=8 / 3, r=120.0$. 


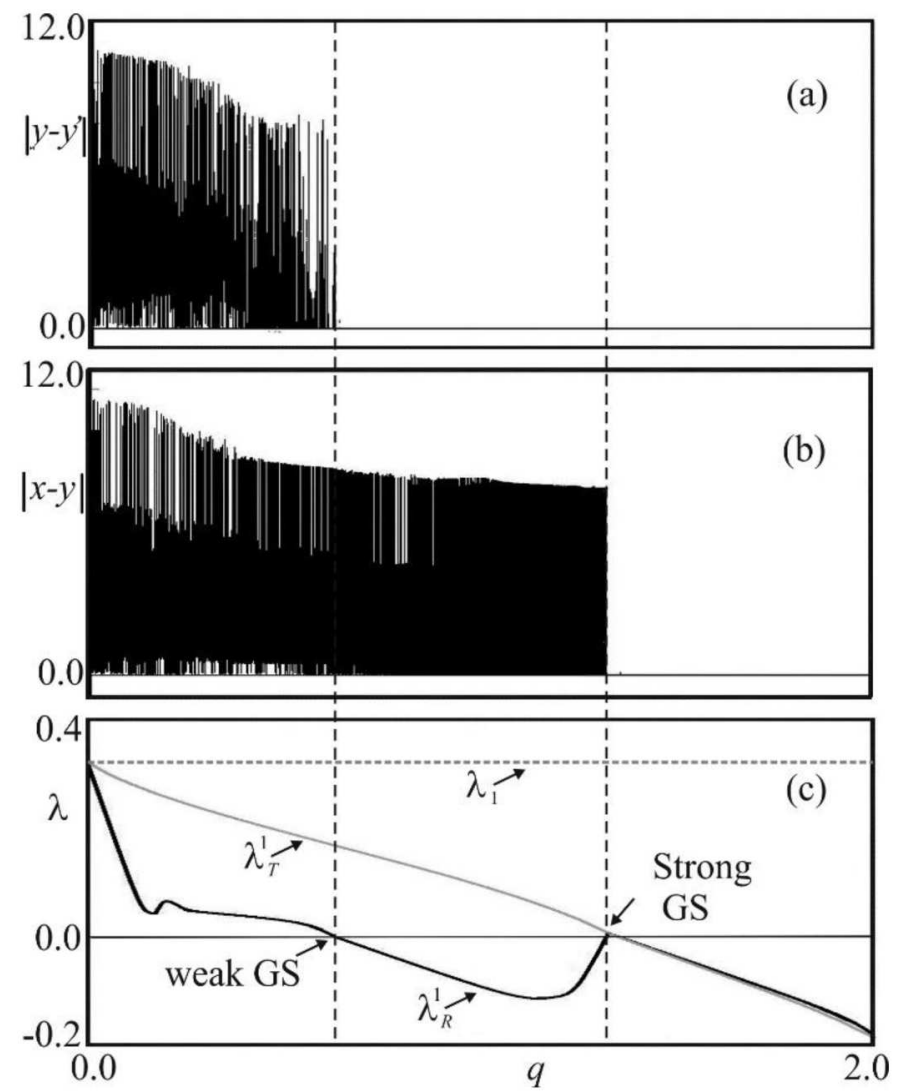

Fig. 4. Bifurcation diagrams of the synchronization error versus the coupling coefficient $q$ in the system of unidirectionally coupled Chua circuits (Eqs. (26a)-(26c)). (a) Identical response oscillators $y-y^{\prime}$, (b) identical drive-response systems $x-y$, (c) corresponding Lyapunov exponents of system (26a)-(26c): the driving LE $\left(\lambda_{1}\right)$ - dashed line, the largest $\operatorname{TLE}\left(\lambda_{T}^{1}\right)$ - gray curve, and the largest RLE $\lambda_{R}^{1}$ - black line. System parameters: $\alpha=8.20$, $\beta=0.85, m_{0}=-0.6724, m_{1}=-1.2464$.

$$
\begin{aligned}
& \dot{y}_{1}^{\prime}=\alpha\left\{y_{2}^{\prime}-y_{1}^{\prime}-\left[m_{0} y_{1}^{\prime}+0.5\left(m_{1}-m_{0}\right)\left(\left|y_{1}^{\prime}+1\right|-\left|y_{1}^{\prime}-1\right|\right)\right]\right\} \\
& \dot{y}_{2}^{\prime}=y_{1}^{\prime}-y_{2}^{\prime}+y_{3}^{\prime}+q\left(x_{2}-y_{2}^{\prime}\right), \\
& \dot{y}_{3}^{\prime}=-\beta y_{2}^{\prime} .
\end{aligned}
$$

The linearized variational equation for calculating the LEs can be written in the following general form:

$$
\begin{aligned}
& \delta \dot{u}_{1}=\alpha\left\{\delta u_{2}-\delta u_{1}-\left[m_{0} \delta u_{1}+0.5\left(m_{1}-m_{0}\right)\left(\frac{x_{1}+1}{\left|x_{1}+1\right|}-\frac{x_{1}-1}{\left|x_{1}-1\right|}\right) \delta u_{1}\right]\right\}, \\
& \delta \dot{u}_{2}=\delta u_{1}-(1+q) \delta u_{2}+\delta u_{3}, \\
& \delta \dot{u}_{3}=-\beta \delta u_{2},
\end{aligned}
$$

where $u=x, y$. For computing TLEs or RLEs, one should assumes $u=x$ or $u=y$, respectively.

Synchronization tendency of unidirectionally coupled Chua circuits system (Eqs. (26a) $-(26 \mathrm{c}))$ is illustrated in Figures $4 \mathrm{a}-4 \mathrm{c}$ and $5 \mathrm{a}-5 \mathrm{c}$. Parameter values adopted in numerical simulations are: $\alpha=8.20, \beta=0.85, m_{0}=-0.6724, m_{1}=-1.2464$, 


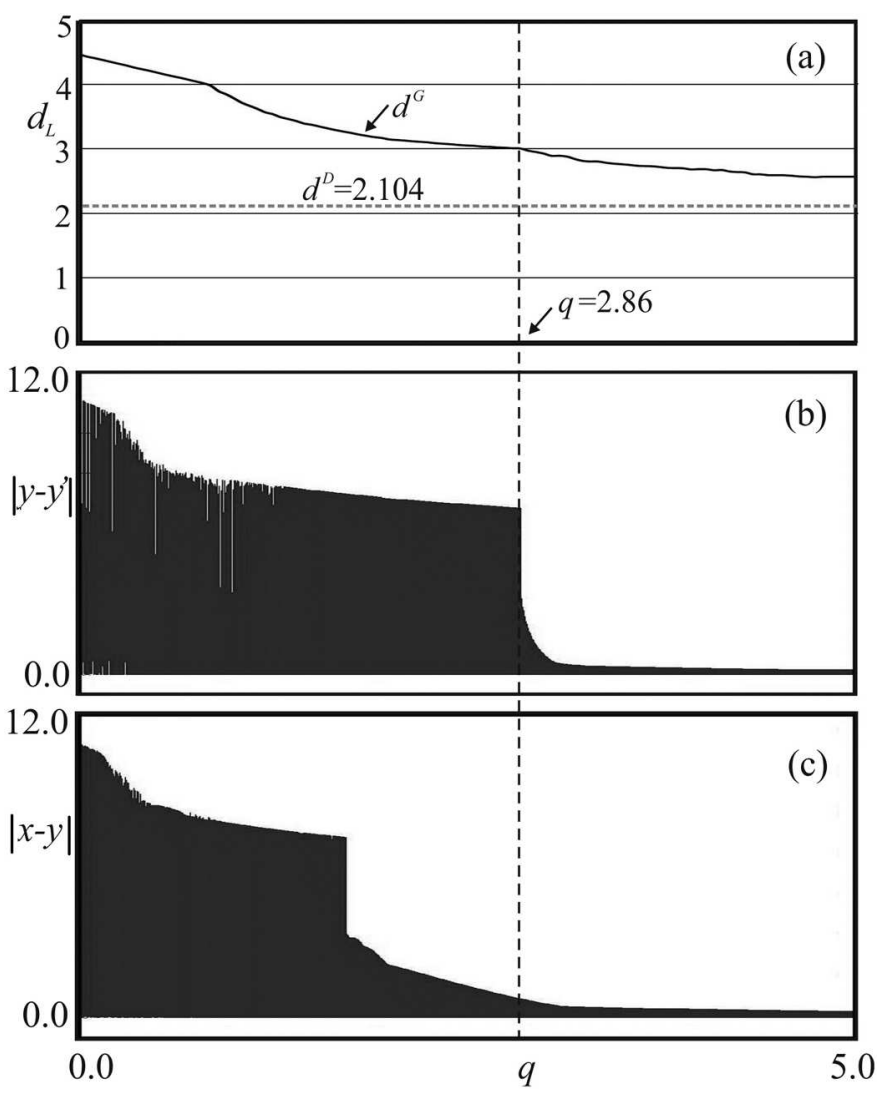

Fig. 5. Bifurcation diagrams versus the coupling coefficient $q$ in the system of unidirectionally coupled Chua circuits (Eqs. (26a)-(26c)): (a) courses Lyapunov dimension of the global $d_{G}$ (black curve) and driving system $d^{D}$ (dashed line in gray) attractors (Eqs. (26a) and (26b)), (b) synchronization error of slightly different response oscillators $y-y^{\prime}\left(\Delta \beta=\beta \times 10^{-4}\right)$ and (c) slightly different drive and response oscillators $x-y$ $\left(\Delta \beta=\beta \times 10^{-4}\right)$. System parameters: $\alpha=8.20, \beta=0.85, m_{0}=-0.6724, m_{1}=-1.2464$.

and coupling strength $q$ is a control parameter. During the computations, trajectories started from the same initial conditions in each bifurcation step: $x_{1}(0)=$ $1.40, x_{2}(0)=0.015, x_{3}(0)=-3.20, y_{1}(0)=2.53, y_{2}(0)=0.012, y_{3}(0)=-3.37$, $y_{1}^{\prime}(0)=2.50, y_{2}^{\prime}(0)=0.01, y_{3}^{\prime}(0)=3.00$. The synchronization error has been computed according to expressions (25a) and (25b). Due to the non-smooth character of the Chua circuit, a method of LEs estimation applicable for such cases has been used [42].

Considering the case of identical oscillators presented in Figures $4 \mathrm{a}-4 \mathrm{c}$ we can notice the same synchronous effects as in above analyzed discrete and continuoustime systems (Eqs. (20a)-(20c) and (24a)-(24c)). Even courses of LEs look similar to these cases. As the coupling parameter increases, one can observe a much greater decrease in the RLE than in the TLE (see Fig. 4c). Hence, the weak GS threshold ( $q=0.62$, Fig. $4 \mathrm{a})$ is over two times lower than the one for the strong GS ( $q=0.1 .31$, Fig. $4 \mathrm{~b})$.

However, the introduction of a slight difference in parameters $\left(\Delta \beta=\beta \times 10^{-4}\right)$ between response oscillators or between the drive and response systems leads to desynchronization also after exceeding the threshold of strong GS determined by a 
negative TLE value. This desynchronous state is illustrated in Figures 5b and 5c. In Figure 5a corresponding course of the global Lyapunov dimension $d^{G}$ of total drive-response system attractor (Eqs. (26a) and (26b)) is shown. Additionally, the dimension of the driving circuit attractor $\left(d^{D}=2.104\right)$ is represented by horizontal dashed line. Both of them have been calculated using the formula given by equation (17). A vertical dashed line in Figures $5 \mathrm{a}-5 \mathrm{c}$ indicate value of the coupling strength $q$ for which the global attractor dimension is equal to $3(q=2.86)$. The comparison of these bifurcation diagrams shows that in both configurations $(x-y$ and $\left.y-y^{\prime}\right)$ investigated systems reach the ICS state when the global attractor dimension is reduced below 3 due to the increase in the coupling parameter. Thus, it can be seen that the ICS threshold is much above the strong GS limit set by negative TLE, although the global attractor dimension is larger than its master system equivalent, i.e., the inequality (15) is fulfilled.

The difference between $x-y$ (Fig. 5c) and $y-y^{\prime}$ (Fig. 5b) plots can result from the specifics of the Chua oscillator, whose trajectory can oscillate around two equivalent critical points. Hence, the possibility of a step change in the amplitude of the synchronization error observed in Figure $5 \mathrm{c}$. The appearance of the ICS seems to be associated with a decrease in $d^{G}$ dimension below 3 for identical systems, i.e. the phase space dimension of the driving system. Such reduction of dimension can diminish the distance between trajectories in the phase space and, as a result, it can lead to the ICS.

\section{Summary and conclusions}

This paper is concentrated on the comparison of the properties (thresholds and stability of synchronization) of weak and strong GS in identical smooth (Lorenz system, logistic map) and non-smooth (Chua oscillator) systems, connected in a master-slave configuration. Analysis of completely identical systems showed that the weak synchronization threshold is lower than the strong one for each of analyzed pairs of coupled oscillators (compare Figs. $2 \mathrm{a}-2 \mathrm{~b}, 3 \mathrm{a}-3 \mathrm{~b}$ and $4 \mathrm{a}-4 \mathrm{~b}$ ). Thus, previous studies have been confirmed and it has been shown that the system's non-smoothness does not affect these thresholds. Moreover, when analyzing the course of Lyapunov exponents (Figs. 2d, 3e and 4c)), their similarity is noticed in all cases under consideration. This fact indicates the same mechanism leading to the strong GS through the weak GS. It can be seen that after exceeding the threshold of strong GS, the RLE and the TLE are practically overlapped. However, this seems to be obvious because in the synchronous state $x=y$ equations (8) and (13) or as a consequence equations (21) and (23) become identical.

On the other hand, the weak GS, characterized by the largest RLE, is extremely sensitive to any perturbations or parameter mismatch. Therefore it is actually unstable. The strong GS is defined by negative TLEs closing the trajectory within synchronization manifold. Such state is robustly stable. Hence, the negative RLEs [condition given by inequality (14)] do not ensure practical synchronization (the ICS) of response subsystems and only the coupling value corresponding to strong synchronization [condition given by inequality (9)] provides it. These properties are characteristic for presented examples of smooth systems (Eqs. (20a)-(20c) and (24a)$(24 \mathrm{c})$ ). In turn, the condition of attractor dimensions equality (Eq. (16)) should provide a strong GS for any case of non-identical (even structurally) oscillators in a master-slave configuration.

However, in the case of coupled non-smooth Chua oscillators with slight parameter mismatch (see Figs. 5a-5c) the ICS does not appear just after crossing the GS threshold, determined by the negative TLE. The results of numerical simulations 
illustrated in Figures 5b and 5c show that the subsystems reach the ICS state only after the coupling factor exceeds the value of $q=2.86$, at which the global attractor dimension is reduced below the value of 3 . Therefore, basing on these results obtained for the case of slightly different oscillators, the strong GS condition can be formulated in form which is intermediate between those given by formulas (9) and (16), i.e.,

$$
d^{G}<d^{P S}
$$

where $d^{P S} \in \mathbb{N}$ is a dimension of the phase space of driving system. Obviously, the above ICS condition should be treated as a weak conjecture, which requires a number of further studies of the GS in non-smooth systems as well as a confirmation that the relationship between the attractor dimension and the ICS, illustrated in Figures $5 \mathrm{a}-5 \mathrm{c}$, is not accidental.

This study has been supported by the Polish National Centre of Science (NCN) under project No. 2017/27/B/ST8/01619.

Publisher's Note The EPJ Publishers remain neutral with regard to jurisdictional claims in published maps and institutional affiliations.

Open Access This is an open access article distributed under the terms of the Creative Commons Attribution License (http://creativecommons.org/licenses/by/4.0), which permits unrestricted use, distribution, and reproduction in any medium, provided the original work is properly cited.

\section{References}

1. C. Huygens, The Pendulum Clock (Iowa State University Press, Ames, 1986)

2. J.W. Rayleigh, in Theory of Sound (Dover Publishing, New York, 1945), p. 480

3. I.I. Blekhman, in Synchronization in Science and Technology (ASME, New York, 1998), p. 350

4. B. Van der Pol, Radio Rev. 1, 701 (1920)

5. J. Hertz, A. Krogh, R. Palmer, in Introduction to the Theory of Neural Computation (Addison-Wesley, Reading, MA, 1991), p. 327

6. H.G. Winful, L. Rahman, Phys. Rev. Lett. 65, 1575 (1990)

7. K. Cuomo, A. Oppenheim, S. Strogatz, IEEE Trans. Circuits Syst. II 40, 626 (1993)

8. Y. Liu, J.R. Rios Leite, Phys. Lett. A, 191, 134 (1994)

9. Y. Soen, N. Cohen, D. Lipson, E. Braun, Phys. Rev. Lett. 82, 3556 (1999)

10. D.J. Watts, in Small Worlds: The Dynamics of Networks Between Order and Randomness (Princeton University Press, Princeton, 1999), p. 262

11. R. Albert, H. Jeong, A.-L. Barabási, Nature (London) 401, 130 (1999)

12. F. Rulkov, M.M. Sushchik, L.S. Tsimring, H.D.I. Abarbanel, Phys. Rev. E 51, 980 (1995)

13. L. Kocarev, U. Parlitz, Phys. Rev. Lett. 74, 1816 (1996)

14. M.G. Rosenblum, A.S. Pikovsky, J. Kurths, Phys. Rev. Lett. 76, 821 (1996)

15. A.S. Pikovsky, M.G. Rosenblum, J. Kurths, IEEE Trans. Circuits Syst. 44, 874 (1997)

16. L-Y. Cao, Y-Ch. Lai, Phys. Rev. E 58, 382 (1998)

17. M.A. Zaks, E.-H. Park, M.G. Rosenblum, J. Kurths, Phys. Rev. Lett. 82, 4228 (1999)

18. M.G. Rosenblum, A.S. Pikovsky, J. Kurths, Phys. Rev. Lett. 78, 4193 (1997)

19. C. Massoler, Phys. Rev. Lett. 86, 2782 (2001)

20. H.U. Voss, Phys. Rev. Lett. 87, 014102 (2001)

21. P. Perlikowski, B. Jagiello, A. Stefanski, T. Kapitaniak, Phys. Rev. E 78, 017203 (2008)

22. H.D.I. Abarbanel, F. Rulkov, M.M. Sushchik, Phys. Rev. E 53, 4528 (1996) 
23. S. Boccaletti, J. Kurths, G. Osipov, D.L. Valladares, C.S. Zhou, Phys. Rep. 366, 1 (2002)

24. P. Perlikowski, A. Stefanski, T. Kapitaniak, J. Sound Vib. 318, 329 (2008)

25. K. Pyragas, Phys. Rev. E 54, 4508 (1996)

26. K. Pyragas, Nonlinear Anal. 3, 29 (1998)

27. M. de Sousa Vieira, A.J. Lichtenberg, Phys. Rev. E 56, 3741 (1997)

28. V.S. Afraimovich, A. Cordonet, F. Rulkov, Phys. Rev. E 66, 016208 (2002)

29. Z. Zheng, X.Wang, M.C. Cross, Phys. Rev. E 65, 056211 (2002)

30. O.I. Moskalenko, A.A. Koronovskii, A.E. Hramov, S. Boccaletti, Phys. Rev. E 86, $036216(2012)$

31. Y.-C. Hung, Y.-T. Huang, M.-C. Ho, C.-K. Hu, Phys. Rev. E 77, 016202 (2008)

32. S. Guan, K. Li, C.-H. Lai, Chaos 16, 023107 (2006)

33. S. Acharyya, R.E. Amritkar, Eur. Phys. J. Special Topics 222, 939 (2013)

34. O.I. Moskalenkoa, A.E. Hramov, A.A. Koronovskii, A.A. Ovchinnikov, Eur. Phys. J. B 82, 69 (2011)

35. A. Yu. Jalnine, Regul. Chaotic Dyn. 18, 214 (2013)

36. J. Yang, G. Hu, Phys. Lett. A 361, 332 (2007)

37. W.K. Wong, B. Zhen, J. Xu, Z. Wang, Chaos 22, 033146 (2012)

38. A.A. Koronovskii, O.I. Moskalenko, S.A. Shurygina, A.E. Hramov, Chaos, Solitons Fractals 46, 12 (2013)

39. J. Kaplan, J. Yorke, Chaotic behavior of multidimensional difference equations, in Functional Differential Equations and Approximation of Fixed Points, edited by Peitgen H.O., Walther H.O. (Springer-Verlag, New York, 1979) pp. 228-237

40. E.N. Lorenz, J. Atmos. Science 20, 130 (1963)

41. L.O. Chua, J. Circuit Syst. Comput. 4, 117 (1994)

42. M. Balcerzak, A. Dabrowski, A. Stefanski, J. Wojewoda, MATEC Web Conf. 148, 10003 (2018) 